\title{
Analysis of Hop-Derived Thiols in Beer Using On-Fiber Derivatization in Combination with HS-SPME and GC-MS/MS
}

Johanna Dennenlöhr, Sarah Thörner, and Nils Rettberg*

Research Institute for Beer and Beverage Analysis, Versuchs- und Lehranstalt für Brauerei in Berlin (VLB) e.V., Seestr. 13, 13353 Berlin, Germany

*Corresponding author. E-mail: n.rettberg@vlb-berlin.org; phone: +49 3045080106.

j.dennenloehr@vlb-berlin.org

s.thoerner@vlb-berlin.org 


\section{Supporting information}

Figure S1. Calibration curves of 4MMP (A), 3MH (B), and 3MHA (C) based on the raw data of Table $\mathbf{2} 2$ used for method validation.

A

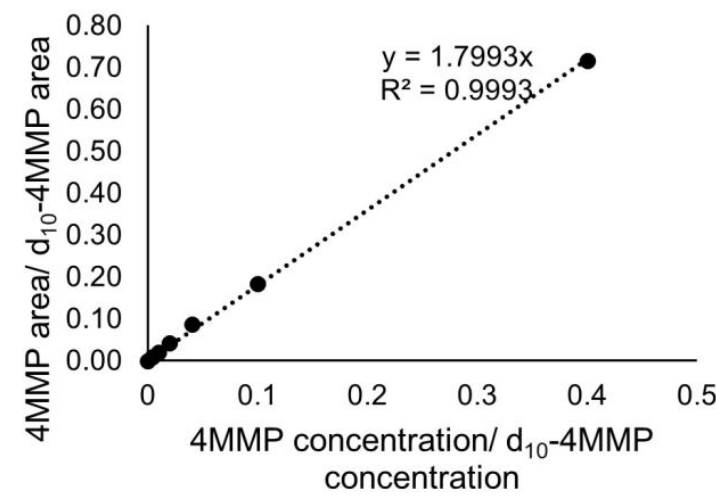

C

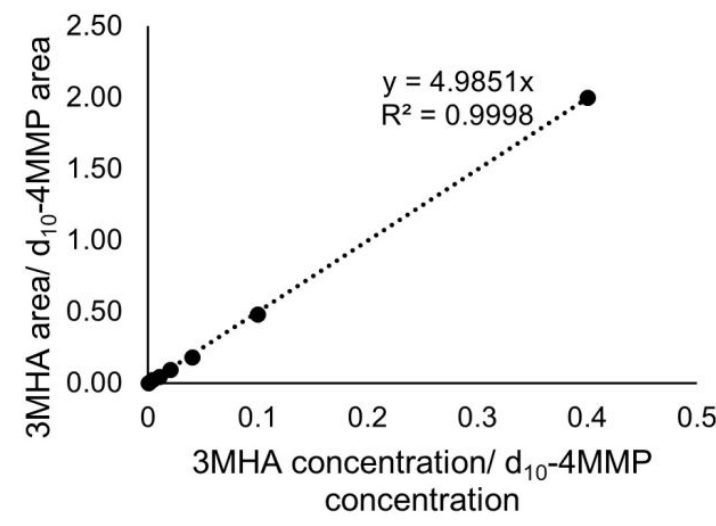

B

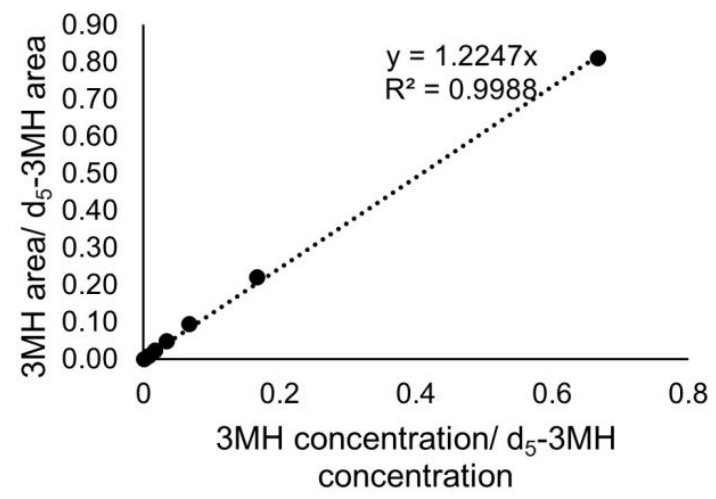




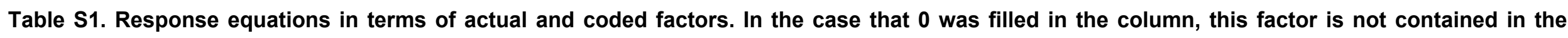
equation.

\begin{tabular}{|c|c|c|c|c|c|c|c|c|c|c|c|c|c|c|c|c|c|c|c|c|}
\hline \multirow[b]{3}{*}{ Factors } & \multicolumn{4}{|c|}{$\mathrm{d}_{10-4 \mathrm{MMP}}$} & \multicolumn{4}{|c|}{ 4MMP } & \multicolumn{4}{|c|}{$d_{5}-3 \mathrm{MH}$} & \multicolumn{4}{|c|}{$3 \mathrm{MH}$} & \multicolumn{4}{|c|}{ 3MHA } \\
\hline & \multicolumn{2}{|c|}{$109 \rightarrow 46$} & \multirow{2}{*}{\multicolumn{2}{|c|}{$322 \rightarrow 46$}} & \multicolumn{2}{|c|}{$99 \rightarrow 43$} & \multicolumn{2}{|c|}{$312 \rightarrow 43$} & \multicolumn{2}{|c|}{$138 \rightarrow 85$} & \multicolumn{2}{|c|}{$319 \rightarrow 87$} & \multicolumn{2}{|c|}{$133 \rightarrow 81$} & \multicolumn{2}{|c|}{$314 \rightarrow 82$} & \multicolumn{2}{|c|}{$115 \rightarrow 81$} & \multicolumn{2}{|c|}{$115 \rightarrow 73$} \\
\hline & actual & coded & & & actual & coded & actual & coded & actual & coded & actual & coded & actual & coded & actual & coded & actual & coded & actual & coded \\
\hline Intercept & -113570 & 26694 & -18085 & 4357 & -79524 & 17282 & -12254 & 3306 & -1849 & 9158 & 2121 & 1435 & -7657 & 14140 & 2565 & 1641 & -637520 & 146400 & $\begin{array}{l}-849346 \\
\end{array}$ & 198300 \\
\hline A & 3321 & $-838,88$ & 674 & $-287,18$ & 1762 & $-1045,3$ & 428 & $-162,89$ & -206 & 1337,01 & -55 & 360,2 & -224 & 2079,28 & -67 & 445,88 & 17247 & 1961,49 & 25144 & 4032,46 \\
\hline B & 0 & 0 & 0 & 0 & 5882 & 330,53 & 773 & $-20,06$ & 2551 & 200,9 & 0 & 0 & 3763 & 240,48 & 0 & 0 & 0 & 0 & 0 & 0 \\
\hline C & 1528 & 3227,46 & 198 & 892,64 & 1212 & 2427,8 & 142 & 590,75 & -445 & 1386,88 & -121 & 367,05 & -693 & 2082,94 & -150 & 452,84 & 4721 & 15735,7 & 7052 & 20770,2 \\
\hline D & 24637 & 2049,48 & 3145 & 570,5 & 17628 & 175,74 & 2457 & 349,35 & 4774 & 955,18 & 814 & 232,79 & 12568 & 1306,03 & 322 & 297,37 & 114155 & 9449,14 & 168074 & 12817,5 \\
\hline $\mathrm{E}$ & 0 & 0 & -109 & 577,97 & 0 & 0 & -87 & 422,67 & 1076 & 0 & 222 & $-16,65$ & 1810 & $-355,18$ & 299 & $-102,93$ & 22763 & -11094 & 19049 & $-1072,9$ \\
\hline $\mathrm{F}$ & 1050 & 5517,99 & 5 & 1840,41 & 932 & 4405,95 & -1 & 1214,3 & 85 & 1815,42 & -68 & 554,87 & 129 & 2698,45 & -77 & 697,78 & 4500 & 22388,5 & 5050 & 30492,8 \\
\hline$A^{*} B$ & 0 & 0 & 0 & 0 & -39 & $-765,27$ & 0 & 0 & 0 & 0 & 0 & 0 & 0 & 0 & 0 & 0 & 0 & 0 & 0 & 0 \\
\hline$A^{*} \mathrm{C}$ & -10 & $-1126,3$ & -3 & $-332,41$ & -10 & $-1177,3$ & -1 & $-177,36$ & 5 & 608,67 & 1 & 163,95 & 8 & 976,75 & 2 & 186,61 & 0 & 0 & 0 & 0 \\
\hline$A^{*} D$ & 0 & 0 & 0 & 0 & -102 & $-834,33$ & 0 & 0 & 60 & 493,23 & 11 & 93,2 & 0 & 0 & 15 & 122,45 & 0 & 0 & 0 & 0 \\
\hline$A^{*} F$ & 0 & 0 & -1 & $-326,28$ & -4 & $-1181,1$ & -1 & $-197,3$ & 4 & 1101,08 & 1 & 326,17 & 5 & 1668,72 & 1 & 405,77 & 0 & 0 & 0 & 0 \\
\hline$C^{*} \mathrm{D}$ & 0 & 0 & 51 & 302,56 & 0 & 0 & 29 & 171,77 & 97 & 573,61 & 20 & 119,36 & 135 & 799,94 & 32 & 187,39 & 0 & 0 & 0 & 0 \\
\hline$C^{*} E$ & 0 & 0 & 4 & 230 & 0 & 0 & 3 & 179,36 & 0 & 0 & 0 & 0 & 0 & 0 & 0 & 0 & -121 & $-7114,3$ & 0 & 0 \\
\hline $\mathrm{C}^{*} \mathrm{~F}$ & 12 & 2634,23 & 4 & 847,59 & 10 & 2208,52 & 3 & 564,83 & 5 & 1123,95 & 1 & 331,95 & 8 & 1727,87 & 2 & 413,02 & 57 & 12623,8 & 77 & 17175,5 \\
\hline$D^{*} \mathrm{~F}$ & 113 & 1725,86 & 36 & 558,31 & 0 & 0 & 22 & 331,39 & 55 & 841,08 & 15 & 228,02 & 80 & 1218,72 & 19 & 295,05 & 514 & 7865,8 & 704 & 10777,7 \\
\hline$E^{\star} F$ & 0 & 0 & 3 & 446,44 & 0 & 0 & 2 & 339,92 & 0 & 0 & 0 & 0 & 0 & 0 & -1 & $-121,64$ & -79 & -12003 & 0 & 0 \\
\hline$A^{2}$ & -32 & $-5289,3$ & -6 & $-983,67$ & -13 & $-2127,9$ & -4 & $-632,59$ & 0 & 0 & 0 & 0 & 0 & 0 & 0 & 0 & -171 & -27914 & -248 & -40545 \\
\hline$B^{2}$ & 0 & 0 & 0 & 0 & -714 & $-1679,2$ & -151 & $-355,38$ & -465 & $-1094,4$ & 0 & 0 & -694 & $-1630,9$ & 0 & 0 & 0 & 0 & 0 & 0 \\
\hline $\mathrm{C}^{2}$ & -41 & $-3510,5$ & -8 & $-645,23$ & -30 & $-2561,5$ & -6 & $-507,66$ & 0 & 0 & 0 & 0 & 0 & 0 & 0 & 0 & -145 & -12486 & -274 & -23494 \\
\hline$D^{2}$ & -13392 & $-5467,3$ & -2388 & $-975,09$ & -6122 & $-2499,2$ & -1694 & $-691,58$ & -5206 & $-2125,2$ & -1020 & $-416,5$ & -8200 & $-3347,6$ & -1006 & $-410,77$ & -61886 & -25265 & -90727 & -37039 \\
\hline$E^{2}$ & 0 & 0 & 0 & 0 & 0 & 0 & 0 & 0 & -54 & $-2170,9$ & -11 & -451 & -93 & $-3751,6$ & -14 & $-558,35$ & -940 & -37990 & -956 & -38645 \\
\hline$F^{2}$ & -12 & $-6749,4$ & 0 & 0 & -7 & $-4284,7$ & 0 & 0 & -3 & $-1949,6$ & 0 & 0 & -5 & $-2955,9$ & 0 & 0 & -44 & -25191 & -60 & -34330 \\
\hline
\end{tabular}


Table S2. Raw data for calibration curves used for method validation.

\begin{tabular}{|c|c|c|c|c|c|c|}
\hline \multirow[t]{6}{*}{ Level 1: } & Compound & $\begin{array}{c}\text { Concentration } \\
{[\mathrm{ng} / \mathrm{L}]}\end{array}$ & $\begin{array}{c}\text { Quantitative } \\
\text { transition }[m / z]\end{array}$ & Area & $\begin{array}{c}\text { Qualitative } \\
\text { transition }[m / z]\end{array}$ & Area \\
\hline & $\mathrm{d}_{10-4 M M P}$ & 250.0 & $109 \rightarrow 46$ & 27213 & $322 \rightarrow 46$ & 10389 \\
\hline & 4MMP & 0.0 & $99 \rightarrow 43$ & 0 & $312 \rightarrow 43$ & 0 \\
\hline & $d_{5}-3 M H$ & 1500.0 & $138 \rightarrow 85$ & 17748 & $319 \rightarrow 87$ & 5863 \\
\hline & $3 \mathrm{MH}$ & 0.0 & $133 \rightarrow 81$ & 0 & $314 \rightarrow 82$ & 0 \\
\hline & $3 \mathrm{MHA}$ & 0.0 & $115 \rightarrow 81$ & 467 & $115 \rightarrow 73$ & 495 \\
\hline \multirow[t]{6}{*}{ Level 2: } & Compound & $\begin{array}{c}\text { Concentration } \\
\text { [ng/L] }\end{array}$ & $\begin{array}{c}\text { Quantitative } \\
\text { transition }[\mathrm{m} / \mathrm{z}]\end{array}$ & Area & $\begin{array}{c}\text { Qualitative } \\
\text { transition }[\mathrm{m} / \mathrm{z}]\end{array}$ & Area \\
\hline & $\mathrm{d}_{10-4 M M P}$ & 250.0 & $109 \rightarrow 46$ & 27664 & $322 \rightarrow 46$ & 10054 \\
\hline & 4MMP & 1.0 & $99 \rightarrow 43$ & 238 & $312 \rightarrow 43$ & 59 \\
\hline & $d_{5}-3 M H$ & 1500.0 & $138 \rightarrow 85$ & 19721 & $319 \rightarrow 87$ & 6450 \\
\hline & $3 \mathrm{MH}$ & 10.0 & $133 \rightarrow 81$ & 192 & $314 \rightarrow 82$ & 41 \\
\hline & 3MHA & 1.0 & $115 \rightarrow 81$ & 1150 & $115 \rightarrow 73$ & 1288 \\
\hline \multirow[t]{6}{*}{ Level 3: } & Compound & $\begin{array}{c}\text { Concentration } \\
{[\mathrm{ng} / \mathrm{L}]}\end{array}$ & $\begin{array}{c}\text { Quantitative } \\
\text { transition }[\mathrm{m} / \mathrm{z}]\end{array}$ & Area & $\begin{array}{c}\text { Qualitative } \\
\text { transition }[\mathrm{m} / \mathrm{z}]\end{array}$ & Area \\
\hline & $\mathrm{d}_{10}-4 \mathrm{MMP}$ & 250.0 & $109 \rightarrow 46$ & 24613 & $322 \rightarrow 46$ & 9209 \\
\hline & 4MMP & 2.5 & $99 \rightarrow 43$ & 493 & $312 \rightarrow 43$ & 126 \\
\hline & $d_{5}-3 \mathrm{MH}$ & 1500.0 & $138 \rightarrow 85$ & 15819 & $319 \rightarrow 87$ & 5218 \\
\hline & $3 \mathrm{MH}$ & 25.0 & $133 \rightarrow 81$ & 378 & $314 \rightarrow 82$ & 86 \\
\hline & $3 \mathrm{MHA}$ & 2.5 & $115 \rightarrow 81$ & 1484 & $115 \rightarrow 73$ & 1573 \\
\hline \multirow[t]{6}{*}{ Level 4: } & Compound & $\begin{array}{c}\text { Concentration } \\
{[\mathrm{ng} / \mathrm{L}]}\end{array}$ & $\begin{array}{c}\text { Quantitative } \\
\text { transition }[\mathrm{m} / \mathrm{z}]\end{array}$ & Area & $\begin{array}{c}\text { Qualitative } \\
\text { transition }[\mathrm{m} / \mathrm{z}]\end{array}$ & Area \\
\hline & $\mathrm{d}_{10-4 M M P}$ & 250.0 & $109 \rightarrow 46$ & 20727 & $322 \rightarrow 46$ & 7602 \\
\hline & 4MMP & 5.0 & $99 \rightarrow 43$ & 884 & $312 \rightarrow 43$ & 220 \\
\hline & $d_{5}-3 M H$ & 1500.0 & $138 \rightarrow 85$ & 12462 & $319 \rightarrow 87$ & 3994 \\
\hline & $3 \mathrm{MH}$ & 50.0 & $133 \rightarrow 81$ & 620 & $314 \rightarrow 82$ & 144 \\
\hline & $3 \mathrm{MHA}$ & 5.0 & $115 \rightarrow 81$ & 2354 & $115 \rightarrow 73$ & 2254 \\
\hline \multirow[t]{6}{*}{ Level 5: } & Compound & $\begin{array}{c}\text { Concentration } \\
{[\mathrm{ng} / \mathrm{L}]}\end{array}$ & $\begin{array}{c}\text { Quantitative } \\
\text { transition }[\mathrm{m} / \mathrm{z}]\end{array}$ & Area & $\begin{array}{c}\text { Qualitative } \\
\text { transition }[\mathrm{m} / \mathrm{z}]\end{array}$ & Area \\
\hline & $\mathrm{d}_{10}-4 \mathrm{MMP}$ & 250.0 & $109 \rightarrow 46$ & 20226 & $322 \rightarrow 46$ & 7311 \\
\hline & 4MMP & 10.0 & $99 \rightarrow 43$ & 1746 & $312 \rightarrow 43$ & 445 \\
\hline & $d_{5}-3 M H$ & 1500.0 & $138 \rightarrow 85$ & 10986 & $319 \rightarrow 87$ & 3588 \\
\hline & $3 \mathrm{MH}$ & 100.0 & $133 \rightarrow 81$ & 1059 & $314 \rightarrow 82$ & 249 \\
\hline & $3 \mathrm{MHA}$ & 10.0 & $115 \rightarrow 81$ & 4036 & $115 \rightarrow 73$ & 4265 \\
\hline \multirow[t]{6}{*}{ Level 6: } & Compound & $\begin{array}{c}\text { Concentration } \\
{[\mathrm{ng} / \mathrm{L}]}\end{array}$ & $\begin{array}{c}\text { Quantitative } \\
\text { transition }[\mathrm{m} / \mathrm{z}]\end{array}$ & Area & $\begin{array}{c}\text { Qualitative } \\
\text { transition }[\mathrm{m} / \mathrm{z}]\end{array}$ & Area \\
\hline & $\mathrm{d}_{10}-4 \mathrm{MMP}$ & 250.0 & $109 \rightarrow 46$ & 20444 & $322 \rightarrow 46$ & 7737 \\
\hline & 4MMP & 25.0 & $99 \rightarrow 43$ & 3764 & $312 \rightarrow 43$ & 932 \\
\hline & $\mathrm{d}_{5}-3 \mathrm{MH}$ & 1500.0 & $138 \rightarrow 85$ & 12100 & $319 \rightarrow 87$ & 3975 \\
\hline & $3 \mathrm{MH}$ & 250.0 & $133 \rightarrow 81$ & 2669 & $314 \rightarrow 82$ & 600 \\
\hline & $3 \mathrm{MHA}$ & 25.0 & $115 \rightarrow 81$ & 10161 & $115 \rightarrow 73$ & 10517 \\
\hline \multirow[t]{6}{*}{ Level 7: } & Compound & $\begin{array}{c}\text { Concentration } \\
\text { [ng/L] }\end{array}$ & $\begin{array}{c}\text { Quantitative } \\
\text { transition }[\mathrm{m} / \mathrm{z}]\end{array}$ & Area & $\begin{array}{c}\text { Qualitative } \\
\text { transition }[\mathrm{m} / \mathrm{z}]\end{array}$ & Area \\
\hline & $\mathrm{d}_{10}-4 \mathrm{MMP}$ & 250.0 & $109 \rightarrow 46$ & 15080 & $322 \rightarrow 46$ & 5581 \\
\hline & 4MMP & 100.0 & $99 \rightarrow 43$ & 10810 & $312 \rightarrow 43$ & 2753 \\
\hline & $d_{5}-3 M H$ & 1500.0 & $138 \rightarrow 85$ & 12448 & $319 \rightarrow 87$ & 4039 \\
\hline & $3 \mathrm{MH}$ & 1000.0 & $133 \rightarrow 81$ & 10087 & $314 \rightarrow 82$ & 2325 \\
\hline & 3MHA & 100.0 & $115 \rightarrow 81$ & 30429 & $115 \rightarrow 73$ & 36078 \\
\hline
\end{tabular}


Table S3. Raw data for recovery experiments used for method validation.

\begin{tabular}{|c|c|c|c|c|c|c|}
\hline \multirow[t]{6}{*}{$\begin{array}{l}\text { Measurement } 1 \text {, } \\
\text { not spiked: }\end{array}$} & Compound & $\begin{array}{c}\text { Concentration } \\
{[\mathrm{ng} / \mathrm{L}]}\end{array}$ & $\begin{array}{c}\text { Quantitative } \\
\text { transition }[\mathrm{m} / \mathrm{z}]\end{array}$ & Area & $\begin{array}{c}\text { Qualitative } \\
\text { transition }[\mathrm{m} / \mathrm{z}]\end{array}$ & Area \\
\hline & 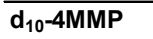 & 250.0 & $109 \rightarrow 46$ & 35276 & $322 \rightarrow 46$ & 10166 \\
\hline & 4MMP & 0.0 & $99 \rightarrow 43$ & 1453 & $312 \rightarrow 43$ & 1557 \\
\hline & $d_{5}-3 \mathrm{MH}$ & 1500.0 & $138 \rightarrow 85$ & 7963 & $319 \rightarrow 87$ & 1887 \\
\hline & $3 \mathrm{MH}$ & 0.0 & $133 \rightarrow 81$ & 1732 & $314 \rightarrow 82$ & 253 \\
\hline & $3 \mathrm{MHA}$ & 0.0 & $115 \rightarrow 81$ & 2457 & $115 \rightarrow 73$ & 1772 \\
\hline \multirow[t]{6}{*}{$\begin{array}{l}\text { Measurement } 2 \text {, } \\
\text { not spiked: }\end{array}$} & Compound & $\begin{array}{c}\text { Concentration } \\
{[\mathrm{ng} / \mathrm{L}]}\end{array}$ & $\begin{array}{c}\text { Quantitative } \\
\text { transition }[\mathrm{m} / \mathrm{z}]\end{array}$ & Area & $\begin{array}{c}\text { Qualitative } \\
\text { transition }[m / z]\end{array}$ & Area \\
\hline & $\overline{d_{10}-4 M M P}$ & 250.0 & $109 \rightarrow 46$ & 27219 & $322 \rightarrow 46$ & 8388 \\
\hline & 4MMP & 0.0 & $99 \rightarrow 43$ & 1367 & $312 \rightarrow 43$ & 1407 \\
\hline & $d_{5}-3 M H$ & 1500.0 & $138 \rightarrow 85$ & 8104 & $319 \rightarrow 87$ & 2041 \\
\hline & $3 \mathrm{MH}$ & 0.0 & $133 \rightarrow 81$ & 1689 & $314 \rightarrow 82$ & 234 \\
\hline & $3 \mathrm{MHA}$ & 0.0 & $115 \rightarrow 81$ & 2333 & $115 \rightarrow 73$ & 1802 \\
\hline \multirow[t]{6}{*}{$\begin{array}{l}\text { Measurement } 3 \text {, } \\
\text { not spiked: }\end{array}$} & Compound & $\begin{array}{c}\text { Concentration } \\
{[\mathrm{ng} / \mathrm{L}]}\end{array}$ & $\begin{array}{c}\text { Quantitative } \\
\text { transition }[\mathrm{m} / \mathrm{z}]\end{array}$ & Area & $\begin{array}{c}\text { Qualitative } \\
\text { transition }[\mathrm{m} / \mathrm{z}]\end{array}$ & Area \\
\hline & $\mathrm{d}_{10-4 M M P}$ & 250.0 & $109 \rightarrow 46$ & 24645 & $322 \rightarrow 46$ & 7535 \\
\hline & 4MMP & 0.0 & $99 \rightarrow 43$ & 1296 & $312 \rightarrow 43$ & 1312 \\
\hline & $d_{5}-3 M H$ & 1500.0 & $138 \rightarrow 85$ & 7128 & $319 \rightarrow 87$ & 1641 \\
\hline & $3 \mathrm{MH}$ & 0.0 & $133 \rightarrow 81$ & 1660 & $314 \rightarrow 82$ & 212 \\
\hline & $3 \mathrm{MHA}$ & 0.0 & $115 \rightarrow 81$ & 1849 & $115 \rightarrow 73$ & 1357 \\
\hline \multirow[t]{6}{*}{$\begin{array}{l}\text { Measurement 1, } \\
\text { spiked, level 1: }\end{array}$} & Compound & $\begin{array}{c}\text { Concentration } \\
{[\mathrm{ng} / \mathrm{L}]}\end{array}$ & $\begin{array}{c}\text { Quantitative } \\
\text { transition }[\mathrm{m} / \mathrm{z}]\end{array}$ & Area & $\begin{array}{c}\text { Qualitative } \\
\text { transition }[\mathrm{m} / \mathrm{z}]\end{array}$ & Area \\
\hline & $\mathrm{d}_{10-4 M M P}$ & 250.0 & $109 \rightarrow 46$ & 23884 & $322 \rightarrow 46$ & 7339 \\
\hline & 4MMP & 5.0 & $99 \rightarrow 43$ & 1891 & $312 \rightarrow 43$ & 1562 \\
\hline & $\mathrm{d}_{5}-3 \mathrm{MH}$ & 1500.0 & $138 \rightarrow 85$ & 6150 & $319 \rightarrow 87$ & 1582 \\
\hline & $3 \mathrm{MH}$ & 50.0 & $133 \rightarrow 81$ & 1720 & $314 \rightarrow 82$ & 257 \\
\hline & $3 \mathrm{MHA}$ & 25.0 & $115 \rightarrow 81$ & 10723 & $115 \rightarrow 73$ & 9371 \\
\hline \multirow[t]{6}{*}{$\begin{array}{l}\text { Measurement 2, } \\
\text { spiked, level 1: }\end{array}$} & Compound & $\begin{array}{c}\text { Concentration } \\
{[\mathrm{ng} / \mathrm{L}]}\end{array}$ & $\begin{array}{c}\text { Quantitative } \\
\text { transition }[\mathrm{m} / \mathrm{z}]\end{array}$ & Area & $\begin{array}{c}\text { Qualitative } \\
\text { transition }[\mathrm{m} / \mathrm{z}]\end{array}$ & Area \\
\hline & 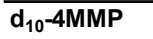 & 250.0 & $109 \rightarrow 46$ & 23657 & $322 \rightarrow 46$ & 7176 \\
\hline & 4MMP & 5.0 & $99 \rightarrow 43$ & 1697 & $312 \rightarrow 43$ & 1233 \\
\hline & $\mathrm{d}_{5}-3 \mathrm{MH}$ & 1500.0 & $138 \rightarrow 85$ & 6714 & $319 \rightarrow 87$ & 1448 \\
\hline & $3 \mathrm{MH}$ & 50.0 & $133 \rightarrow 81$ & 1842 & $314 \rightarrow 82$ & 237 \\
\hline & $3 \mathrm{MHA}$ & 25.0 & $115 \rightarrow 81$ & 11156 & $115 \rightarrow 73$ & 9933 \\
\hline \multirow[t]{6}{*}{$\begin{array}{l}\text { Measurement 3, } \\
\text { spiked, level 1: }\end{array}$} & Compound & $\begin{array}{c}\text { Concentration } \\
{[\mathrm{ng} / \mathrm{L}]}\end{array}$ & $\begin{array}{c}\text { Quantitative } \\
\text { transition }[\mathrm{m} / \mathrm{z}]\end{array}$ & Area & $\begin{array}{c}\text { Qualitative } \\
\text { transition }[\mathrm{m} / \mathrm{z}]\end{array}$ & Area \\
\hline & $\mathrm{d}_{10-4 \mathrm{MMP}}$ & 250.0 & $109 \rightarrow 46$ & 19705 & $322 \rightarrow 46$ & 5877 \\
\hline & 4MMP & 5.0 & $99 \rightarrow 43$ & 1877 & $312 \rightarrow 43$ & 1056 \\
\hline & $\mathrm{d}_{5}-3 \mathrm{MH}$ & 1500.0 & $138 \rightarrow 85$ & 7028 & $319 \rightarrow 87$ & 1381 \\
\hline & $3 \mathrm{MH}$ & 50.0 & $133 \rightarrow 81$ & 1665 & $314 \rightarrow 82$ & 326 \\
\hline & $3 \mathrm{MHA}$ & 25.0 & $115 \rightarrow 81$ & 11081 & $115 \rightarrow 73$ & 9917 \\
\hline \multirow[t]{6}{*}{$\begin{array}{l}\text { Measurement 1, } \\
\text { spiked, level 2: }\end{array}$} & Compound & $\begin{array}{c}\text { Concentration } \\
\text { [ng/L] }\end{array}$ & $\begin{array}{c}\text { Quantitative } \\
\text { transition }[\mathrm{m} / \mathrm{z}]\end{array}$ & Area & $\begin{array}{c}\text { Qualitative } \\
\text { transition }[\mathrm{m} / \mathrm{z}]\end{array}$ & Area \\
\hline & $\mathrm{d}_{10}-4 \mathrm{MMP}$ & 250.0 & $109 \rightarrow 46$ & 16730 & $322 \rightarrow 46$ & 5660 \\
\hline & 4MMP & 25.0 & $99 \rightarrow 43$ & 3656 & $312 \rightarrow 43$ & 1760 \\
\hline & $d_{5}-3 \mathrm{MH}$ & 1500.0 & $138 \rightarrow 85$ & 4893 & $319 \rightarrow 87$ & 1569 \\
\hline & $3 \mathrm{MH}$ & 250.0 & $133 \rightarrow 81$ & 2295 & $314 \rightarrow 82$ & 341 \\
\hline & $3 \mathrm{MHA}$ & 125.0 & $115 \rightarrow 81$ & 36950 & $115 \rightarrow 73$ & 33149 \\
\hline \multirow[t]{6}{*}{$\begin{array}{l}\text { Measurement 2, } \\
\text { spiked, level 2: }\end{array}$} & Compound & $\begin{array}{c}\text { Concentration } \\
\text { [ng/L] }\end{array}$ & $\begin{array}{c}\text { Quantitative } \\
\text { transition }[\mathrm{m} / \mathrm{z}]\end{array}$ & Area & $\begin{array}{c}\text { Qualitative } \\
\text { transition }[\mathrm{m} / \mathrm{z}]\end{array}$ & Area \\
\hline & $\mathrm{d}_{10-4 M M P}$ & 250.0 & $109 \rightarrow 46$ & 17789 & $322 \rightarrow 46$ & 5428 \\
\hline & 4MMP & 25.0 & $99 \rightarrow 43$ & 3559 & $312 \rightarrow 43$ & 1681 \\
\hline & $d_{5}-3 \mathrm{MH}$ & 1500.0 & $138 \rightarrow 85$ & 5798 & $319 \rightarrow 87$ & 1100 \\
\hline & $3 \mathrm{MH}$ & 250.0 & $133 \rightarrow 81$ & 2251 & $314 \rightarrow 82$ & 347 \\
\hline & $3 \mathrm{MHA}$ & 125.0 & $115 \rightarrow 81$ & 41919 & $115 \rightarrow 73$ & 38758 \\
\hline \multirow[t]{6}{*}{$\begin{array}{l}\text { Measurement 3, } \\
\text { spiked, level 2: }\end{array}$} & Compound & $\begin{array}{c}\text { Concentration } \\
{[\mathrm{ng} / \mathrm{L}]}\end{array}$ & $\begin{array}{c}\text { Quantitative } \\
\text { transition }[\mathrm{m} / \mathrm{z}]\end{array}$ & Area & $\begin{array}{c}\text { Qualitative } \\
\text { transition }[\mathrm{m} / \mathrm{z}]\end{array}$ & Area \\
\hline & $\mathrm{d}_{10-4 M M P}$ & 250.0 & $109 \rightarrow 46$ & 15638 & $322 \rightarrow 46$ & 4851 \\
\hline & 4MMP & 25.0 & $99 \rightarrow 43$ & 3022 & $312 \rightarrow 43$ & 1562 \\
\hline & $d_{5}-3 M H$ & 1500.0 & $138 \rightarrow 85$ & 6503 & $319 \rightarrow 87$ & 1473 \\
\hline & $3 \mathrm{MH}$ & 250.0 & $133 \rightarrow 81$ & 2166 & $314 \rightarrow 82$ & 337 \\
\hline & $3 \mathrm{MHA}$ & 125.0 & $115 \rightarrow 81$ & 42924 & $115 \rightarrow 73$ & 39671 \\
\hline
\end{tabular}


Table S4. Raw data for measurement precision used for method validation.

\begin{tabular}{|c|c|c|c|c|c|}
\hline \multirow[t]{6}{*}{ Measurement 1: } & Compound & $\begin{array}{c}\text { Quantitative } \\
\text { transition }[\mathrm{m} / \mathrm{z}]\end{array}$ & Area & $\begin{array}{c}\text { Qualitative } \\
\text { transition }[\mathrm{m} / \mathrm{z}]\end{array}$ & Area \\
\hline & $\overline{d_{10}-4 M M P}$ & $109 \rightarrow 46$ & 17789 & $322 \rightarrow 46$ & 5428 \\
\hline & 4MMP & $99 \rightarrow 43$ & 3559 & $312 \rightarrow 43$ & 1681 \\
\hline & $\mathrm{d}_{5}-3 \mathrm{MH}$ & $138 \rightarrow 85$ & 5798 & $319 \rightarrow 87$ & 1100 \\
\hline & $3 \mathrm{MH}$ & $133 \rightarrow 81$ & 2251 & $314 \rightarrow 82$ & 347 \\
\hline & $3 \mathrm{MHA}$ & $115 \rightarrow 81$ & 41919 & $115 \rightarrow 73$ & 38758 \\
\hline \multirow[t]{6}{*}{ Measurement 2: } & Compound & $\begin{array}{c}\text { Quantitative } \\
\text { transition }[\mathrm{m} / \mathrm{z}]\end{array}$ & Area & $\begin{array}{c}\text { Qualitative } \\
\text { transition }[\mathrm{m} / \mathrm{z}]\end{array}$ & Area \\
\hline & $\overline{d_{10}-4 M M P}$ & $109 \rightarrow 46$ & 15638 & $322 \rightarrow 46$ & 4851 \\
\hline & $4 \mathrm{MMP}$ & $99 \rightarrow 43$ & 3022 & $312 \rightarrow 43$ & 1562 \\
\hline & $d_{5}-3 M H$ & $138 \rightarrow 85$ & 6503 & $319 \rightarrow 87$ & 1473 \\
\hline & $3 \mathrm{MH}$ & $133 \rightarrow 81$ & 2166 & $314 \rightarrow 82$ & 337 \\
\hline & $3 \mathrm{MHA}$ & $115 \rightarrow 81$ & 42924 & $115 \rightarrow 73$ & 39671 \\
\hline \multirow[t]{6}{*}{ Measurement 3: } & Compound & $\begin{array}{c}\text { Quantitative } \\
\text { transition }[\mathrm{m} / \mathrm{z}]\end{array}$ & Area & $\begin{array}{c}\text { Qualitative } \\
\text { transition }[\mathrm{m} / \mathrm{z}]\end{array}$ & Area \\
\hline & $\overline{d_{10}-4 M M P}$ & $109 \rightarrow 46$ & 14764 & $322 \rightarrow 46$ & 4336 \\
\hline & 4MMP & $99 \rightarrow 43$ & 2311 & $312 \rightarrow 43$ & 980 \\
\hline & $d_{5}-3 M H$ & $138 \rightarrow 85$ & 5136 & $319 \rightarrow 87$ & 1195 \\
\hline & $3 \mathrm{MH}$ & $133 \rightarrow 81$ & 2118 & $314 \rightarrow 82$ & 315 \\
\hline & $3 \mathrm{MHA}$ & $115 \rightarrow 81$ & 38112 & $115 \rightarrow 73$ & 34394 \\
\hline \multirow[t]{6}{*}{ Measurement 4: } & Compound & $\begin{array}{c}\text { Quantitative } \\
\text { transition }[\mathrm{m} / \mathrm{z}]\end{array}$ & Area & $\begin{array}{c}\text { Qualitative } \\
\text { transition }[\mathrm{m} / \mathrm{z}]\end{array}$ & Area \\
\hline & $\overline{d_{10}-4 M M P}$ & $109 \rightarrow 46$ & 11795 & $322 \rightarrow 46$ & 3776 \\
\hline & 4MMP & $99 \rightarrow 43$ & 2439 & $312 \rightarrow 43$ & 1283 \\
\hline & $d_{5}-3 M H$ & $138 \rightarrow 85$ & 4152 & $319 \rightarrow 87$ & 944 \\
\hline & $3 \mathrm{MH}$ & $133 \rightarrow 81$ & 1654 & $314 \rightarrow 82$ & 245 \\
\hline & $3 \mathrm{MHA}$ & $115 \rightarrow 81$ & 26805 & $115 \rightarrow 73$ & 25193 \\
\hline \multirow[t]{6}{*}{ Measurement 5: } & Compound & $\begin{array}{c}\text { Quantitative } \\
\text { transition }[\mathrm{m} / \mathrm{z}]\end{array}$ & Area & $\begin{array}{c}\text { Qualitative } \\
\text { transition }[\mathrm{m} / \mathrm{z}]\end{array}$ & Area \\
\hline & $\mathrm{d}_{10-4 M M P}$ & $109 \rightarrow 46$ & 10862 & $322 \rightarrow 46$ & 3541 \\
\hline & $4 \mathrm{MMP}$ & $99 \rightarrow 43$ & 2293 & $312 \rightarrow 43$ & 904 \\
\hline & $d_{5}-3 M H$ & $138 \rightarrow 85$ & 4418 & $319 \rightarrow 87$ & 840 \\
\hline & $3 \mathrm{MH}$ & $133 \rightarrow 81$ & 1552 & $314 \rightarrow 82$ & 234 \\
\hline & $3 \mathrm{MHA}$ & $115 \rightarrow 81$ & 27937 & $115 \rightarrow 73$ & 24505 \\
\hline \multirow[t]{6}{*}{ Measurement 6: } & Compound & $\begin{array}{c}\text { Quantitative } \\
\text { transition }[\mathrm{m} / \mathrm{z}]\end{array}$ & Area & $\begin{array}{c}\text { Qualitative } \\
\text { transition }[\mathrm{m} / \mathrm{z}]\end{array}$ & Area \\
\hline & $\mathrm{d}_{10-4 M M P}$ & $109 \rightarrow 46$ & 12064 & $322 \rightarrow 46$ & 3882 \\
\hline & $4 \mathrm{MMP}$ & $99 \rightarrow 43$ & 2475 & $312 \rightarrow 43$ & 1283 \\
\hline & $d_{5}-3 M H$ & $138 \rightarrow 85$ & 5392 & $319 \rightarrow 87$ & 1116 \\
\hline & $3 \mathrm{MH}$ & $133 \rightarrow 81$ & 1653 & $314 \rightarrow 82$ & 247 \\
\hline & $3 \mathrm{MHA}$ & $115 \rightarrow 81$ & 31609 & $115 \rightarrow 73$ & 28418 \\
\hline
\end{tabular}


Table S5. Raw data for ruggedness regarding elapsed assay times used for method validation.

\begin{tabular}{|c|c|c|c|c|c|}
\hline \multirow[t]{6}{*}{$\begin{array}{l}\text { Beginning, } \\
\text { measurement } 1 \text { : }\end{array}$} & Compound & $\begin{array}{c}\text { Quantitative } \\
\text { transition }[\mathrm{m} / \mathrm{z}]\end{array}$ & Area & $\begin{array}{c}\text { Qualitative } \\
\text { transition }[\mathrm{m} / \mathrm{z}]\end{array}$ & Area \\
\hline & $\mathrm{d}_{10^{-}-4 \mathrm{MMP}}$ & $109 \rightarrow 46$ & 17540 & $322 \rightarrow 46$ & 5505 \\
\hline & $4 \mathrm{MMP}$ & $99 \rightarrow 43$ & 2933 & $312 \rightarrow 43$ & 1414 \\
\hline & $d_{5}-3 M H$ & $138 \rightarrow 85$ & 4880 & $319 \rightarrow 87$ & 1208 \\
\hline & $3 \mathrm{MH}$ & $133 \rightarrow 81$ & 1641 & $314 \rightarrow 82$ & 264 \\
\hline & $3 \mathrm{MHA}$ & $115 \rightarrow 81$ & 47214 & $115 \rightarrow 73$ & 43183 \\
\hline \multirow[t]{6}{*}{$\begin{array}{l}\text { Beginning, } \\
\text { measurement 2: }\end{array}$} & Compound & $\begin{array}{c}\text { Quantitative } \\
\text { transition }[\mathrm{m} / \mathrm{z}]\end{array}$ & Area & $\begin{array}{c}\text { Qualitative } \\
\text { transition }[\mathrm{m} / \mathrm{z}]\end{array}$ & Area \\
\hline & $\overline{d_{10}-4 M M P}$ & $109 \rightarrow 46$ & 24448 & $322 \rightarrow 46$ & 7265 \\
\hline & $4 \mathrm{MMP}$ & $99 \rightarrow 43$ & 4651 & $312 \rightarrow 43$ & 2618 \\
\hline & $d_{5}-3 M H$ & $138 \rightarrow 85$ & 8338 & $319 \rightarrow 87$ & 1916 \\
\hline & $3 \mathrm{MH}$ & $133 \rightarrow 81$ & 3101 & $314 \rightarrow 82$ & 489 \\
\hline & $3 \mathrm{MHA}$ & $115 \rightarrow 81$ & 70993 & $115 \rightarrow 73$ & 65668 \\
\hline \multirow[t]{6}{*}{$\begin{array}{l}\text { Middle, } \\
\text { measurement } 1 \text { : }\end{array}$} & Compound & $\begin{array}{c}\text { Quantitative } \\
\text { transition }[m / z]\end{array}$ & Area & $\begin{array}{c}\text { Qualitative } \\
\text { transition }[\mathrm{m} / \mathrm{z}]\end{array}$ & Area \\
\hline & $\mathrm{d}_{10-4 M M P}$ & $109 \rightarrow 46$ & 18483 & $322 \rightarrow 46$ & 5906 \\
\hline & $4 \mathrm{MMP}$ & $99 \rightarrow 43$ & 3030 & $312 \rightarrow 43$ & 1673 \\
\hline & $d_{5}-3 M H$ & $138 \rightarrow 85$ & 6463 & $319 \rightarrow 87$ & 1557 \\
\hline & $3 \mathrm{MH}$ & $133 \rightarrow 81$ & 2580 & $314 \rightarrow 82$ & 397 \\
\hline & $3 \mathrm{MHA}$ & $115 \rightarrow 81$ & 55321 & $115 \rightarrow 73$ & 51934 \\
\hline \multirow[t]{6}{*}{$\begin{array}{l}\text { Middle, } \\
\text { measurement 2: }\end{array}$} & Compound & $\begin{array}{c}\text { Quantitative } \\
\text { transition }[m / z]\end{array}$ & Area & $\begin{array}{c}\text { Qualitative } \\
\text { transition }[\mathrm{m} / \mathrm{z}]\end{array}$ & Area \\
\hline & $\mathrm{d}_{10^{-}-4 M M P}$ & $109 \rightarrow 46$ & 17653 & $322 \rightarrow 46$ & 5680 \\
\hline & 4MMP & $99 \rightarrow 43$ & 2739 & $312 \rightarrow 43$ & 1585 \\
\hline & $d_{5}-3 M H$ & $138 \rightarrow 85$ & 6665 & $319 \rightarrow 87$ & 2009 \\
\hline & $3 \mathrm{MH}$ & $133 \rightarrow 81$ & 2733 & $314 \rightarrow 82$ & 421 \\
\hline & $3 \mathrm{MHA}$ & $115 \rightarrow 81$ & 52739 & $115 \rightarrow 73$ & 47149 \\
\hline \multirow[t]{6}{*}{$\begin{array}{l}\text { End, } \\
\text { measurement } 1 \text { : }\end{array}$} & Compound & $\begin{array}{c}\text { Quantitative } \\
\text { transition }[m / z]\end{array}$ & Area & $\begin{array}{c}\text { Qualitative } \\
\text { transition }[\mathrm{m} / \mathrm{z}]\end{array}$ & Area \\
\hline & $\mathrm{d}_{10^{-}-4 M M P}$ & $109 \rightarrow 46$ & 11573 & $322 \rightarrow 46$ & 3421 \\
\hline & $4 \mathrm{MMP}$ & $99 \rightarrow 43$ & 2318 & $312 \rightarrow 43$ & 1024 \\
\hline & $d_{5}-3 \mathrm{MH}$ & $138 \rightarrow 85$ & 4009 & $319 \rightarrow 87$ & 849 \\
\hline & $3 \mathrm{MH}$ & $133 \rightarrow 81$ & 1617 & $314 \rightarrow 82$ & 247 \\
\hline & $3 \mathrm{MHA}$ & $115 \rightarrow 81$ & 29399 & $115 \rightarrow 73$ & 26301 \\
\hline \multirow[t]{6}{*}{$\begin{array}{l}\text { End, } \\
\text { measurement 2: }\end{array}$} & Compound & $\begin{array}{c}\text { Quantitative } \\
\text { transition }[\mathrm{m} / \mathrm{z}]\end{array}$ & Area & $\begin{array}{c}\text { Qualitative } \\
\text { transition }[m / z]\end{array}$ & Area \\
\hline & $\overline{d_{10}-4 M M P}$ & $109 \rightarrow 46$ & 13603 & $322 \rightarrow 46$ & 4030 \\
\hline & $4 \mathrm{MMP}$ & $99 \rightarrow 43$ & 2572 & $312 \rightarrow 43$ & 1503 \\
\hline & $d_{5}-3 M H$ & $138 \rightarrow 85$ & 4352 & $319 \rightarrow 87$ & 1066 \\
\hline & $3 \mathrm{MH}$ & $133 \rightarrow 81$ & 1860 & $314 \rightarrow 82$ & 265 \\
\hline & $3 \mathrm{MHA}$ & $115 \rightarrow 81$ & 34137 & $115 \rightarrow 73$ & 30359 \\
\hline
\end{tabular}


Table S6. Raw data for ruggedness regarding different days used for method validation.

\begin{tabular}{|c|c|c|c|c|c|}
\hline \multirow[t]{6}{*}{$\begin{array}{l}\text { Day 1, } \\
\text { measurement 1: }\end{array}$} & Compound & $\begin{array}{c}\text { Quantitative } \\
\text { transition }[\mathrm{m} / \mathrm{z}]\end{array}$ & Area & $\begin{array}{c}\text { Qualitative } \\
\text { transition }[\mathrm{m} / \mathrm{z}]\end{array}$ & Area \\
\hline & $\overline{d_{10-4 M M P}}$ & $109 \rightarrow 46$ & 17540 & $322 \rightarrow 46$ & 5505 \\
\hline & 4MMP & $99 \rightarrow 43$ & 2933 & $312 \rightarrow 43$ & 1414 \\
\hline & $d_{5}-3 M H$ & $138 \rightarrow 85$ & 4880 & $319 \rightarrow 87$ & 1208 \\
\hline & $3 \mathrm{MH}$ & $133 \rightarrow 81$ & 1641 & $314 \rightarrow 82$ & 264 \\
\hline & $3 \mathrm{MHA}$ & $115 \rightarrow 81$ & 47214 & $115 \rightarrow 73$ & 43183 \\
\hline \multirow[t]{6}{*}{$\begin{array}{l}\text { Day } 1 \text {, } \\
\text { measurement 2: }\end{array}$} & Compound & $\begin{array}{c}\text { Quantitative } \\
\text { transition }[\mathrm{m} / \mathrm{z}]\end{array}$ & Area & $\begin{array}{c}\text { Qualitative } \\
\text { transition }[\mathrm{m} / \mathrm{z}]\end{array}$ & Area \\
\hline & $\mathrm{d}_{10-4 M M P}$ & $109 \rightarrow 46$ & 18483 & $322 \rightarrow 46$ & 5906 \\
\hline & 4MMP & $99 \rightarrow 43$ & 3030 & $312 \rightarrow 43$ & 1673 \\
\hline & $d_{5}-3 M H$ & $138 \rightarrow 85$ & 6463 & $319 \rightarrow 87$ & 1557 \\
\hline & $3 \mathrm{MH}$ & $133 \rightarrow 81$ & 2580 & $314 \rightarrow 82$ & 397 \\
\hline & $3 \mathrm{MHA}$ & $115 \rightarrow 81$ & 55321 & $115 \rightarrow 73$ & 51934 \\
\hline \multirow[t]{6}{*}{$\begin{array}{l}\text { Day 1, } \\
\text { measurement 3: }\end{array}$} & Compound & $\begin{array}{c}\text { Quantitative } \\
\text { transition }[\mathrm{m} / \mathrm{z}]\end{array}$ & Area & $\begin{array}{c}\text { Qualitative } \\
\text { transition }[\mathrm{m} / \mathrm{z}]\end{array}$ & Area \\
\hline & $\overline{d_{10-4 M M P}}$ & $109 \rightarrow 46$ & 17653 & $322 \rightarrow 46$ & 5680 \\
\hline & $4 \mathrm{MMP}$ & $99 \rightarrow 43$ & 2739 & $312 \rightarrow 43$ & 1585 \\
\hline & $d_{5}-3 M H$ & $138 \rightarrow 85$ & 6665 & $319 \rightarrow 87$ & 2009 \\
\hline & $3 \mathrm{MH}$ & $133 \rightarrow 81$ & 2733 & $314 \rightarrow 82$ & 421 \\
\hline & $3 \mathrm{MHA}$ & $115 \rightarrow 81$ & 52739 & $115 \rightarrow 73$ & 47149 \\
\hline \multirow[t]{6}{*}{$\begin{array}{l}\text { Day 2, } \\
\text { measurement 1: }\end{array}$} & Compound & $\begin{array}{c}\text { Quantitative } \\
\text { transition }[\mathrm{m} / \mathrm{z}]\end{array}$ & Area & $\begin{array}{c}\text { Qualitative } \\
\text { transition }[\mathrm{m} / \mathrm{z}]\end{array}$ & Area \\
\hline & $\overline{d_{10-4 M M P}}$ & $109 \rightarrow 46$ & 16730 & $322 \rightarrow 46$ & 5660 \\
\hline & 4MMP & $99 \rightarrow 43$ & 3656 & $312 \rightarrow 43$ & 1760 \\
\hline & $d_{5}-3 M H$ & $138 \rightarrow 85$ & 4893 & $319 \rightarrow 87$ & 1569 \\
\hline & $3 \mathrm{MH}$ & $133 \rightarrow 81$ & 2295 & $314 \rightarrow 82$ & 341 \\
\hline & $3 \mathrm{MHA}$ & $115 \rightarrow 81$ & 36950 & $115 \rightarrow 73$ & 33149 \\
\hline \multirow[t]{6}{*}{$\begin{array}{l}\text { Day 2, } \\
\text { measurement 2: }\end{array}$} & Compound & $\begin{array}{c}\text { Quantitative } \\
\text { transition }[\mathrm{m} / \mathrm{z}]\end{array}$ & Area & $\begin{array}{c}\text { Qualitative } \\
\text { transition }[\mathrm{m} / \mathrm{z}]\end{array}$ & Area \\
\hline & $\overline{d_{10-4 M M P}}$ & $109 \rightarrow 46$ & 17789 & $322 \rightarrow 46$ & 5428 \\
\hline & 4MMP & $99 \rightarrow 43$ & 3559 & $312 \rightarrow 43$ & 1681 \\
\hline & $d_{5}-3 M H$ & $138 \rightarrow 85$ & 5798 & $319 \rightarrow 87$ & 1100 \\
\hline & $3 \mathrm{MH}$ & $133 \rightarrow 81$ & 2251 & $314 \rightarrow 82$ & 347 \\
\hline & $3 \mathrm{MHA}$ & $115 \rightarrow 81$ & 41919 & $115 \rightarrow 73$ & 38758 \\
\hline \multirow[t]{6}{*}{$\begin{array}{l}\text { Day 2, } \\
\text { measurement 3: }\end{array}$} & Compound & $\begin{array}{c}\text { Quantitative } \\
\text { transition }[\mathrm{m} / \mathrm{z}]\end{array}$ & Area & $\begin{array}{c}\text { Qualitative } \\
\text { transition }[\mathrm{m} / \mathrm{z}]\end{array}$ & Area \\
\hline & $\overline{d_{10-4 M M P}}$ & $109 \rightarrow 46$ & 15638 & $322 \rightarrow 46$ & 4851 \\
\hline & 4MMP & $99 \rightarrow 43$ & 3022 & $312 \rightarrow 43$ & 1562 \\
\hline & $d_{5}-3 M H$ & $138 \rightarrow 85$ & 6503 & $319 \rightarrow 87$ & 1473 \\
\hline & $3 \mathrm{MH}$ & $133 \rightarrow 81$ & 2166 & $314 \rightarrow 82$ & 337 \\
\hline & $3 \mathrm{MHA}$ & $115 \rightarrow 81$ & 42924 & $115 \rightarrow 73$ & 39671 \\
\hline
\end{tabular}


Table S7. Raw data for ruggedness regarding different analysts used for method validation.

\begin{tabular}{|c|c|c|c|c|c|}
\hline \multirow[t]{6}{*}{$\begin{array}{l}\text { Analyst 1, } \\
\text { measurement 1: }\end{array}$} & Compound & $\begin{array}{c}\text { Quantitative } \\
\text { transition }[\mathrm{m} / \mathrm{z}]\end{array}$ & Area & $\begin{array}{c}\text { Qualitative } \\
\text { transition }[\mathrm{m} / \mathrm{z}]\end{array}$ & Area \\
\hline & $\mathrm{d}_{10}-4 \mathrm{MMP}$ & $109 \rightarrow 46$ & 24417 & $322 \rightarrow 46$ & 7386 \\
\hline & $4 \mathrm{MMP}$ & $99 \rightarrow 43$ & 2610 & $312 \rightarrow 43$ & 1578 \\
\hline & $d_{5}-3 M H$ & $138 \rightarrow 85$ & 8258 & $319 \rightarrow 87$ & 2052 \\
\hline & $3 \mathrm{MH}$ & $133 \rightarrow 81$ & 1810 & $314 \rightarrow 82$ & 301 \\
\hline & $3 \mathrm{MHA}$ & $115 \rightarrow 81$ & 15977 & $115 \rightarrow 73$ & 14750 \\
\hline \multirow[t]{6}{*}{$\begin{array}{l}\text { Analyst 1, } \\
\text { measurement 2: }\end{array}$} & Compound & $\begin{array}{c}\text { Quantitative } \\
\text { transition }[\mathrm{m} / \mathrm{z}]\end{array}$ & Area & $\begin{array}{c}\text { Qualitative } \\
\text { transition }[\mathrm{m} / \mathrm{z}]\end{array}$ & Area \\
\hline & $\mathrm{d}_{10}-4 \mathrm{MMP}$ & $109 \rightarrow 46$ & 25166 & $322 \rightarrow 46$ & 7205 \\
\hline & 4MMP & $99 \rightarrow 43$ & 2296 & $312 \rightarrow 43$ & 1119 \\
\hline & $d_{5}-3 M H$ & $138 \rightarrow 85$ & 8090 & $319 \rightarrow 87$ & 2002 \\
\hline & $3 \mathrm{MH}$ & $133 \rightarrow 81$ & 1905 & $314 \rightarrow 82$ & 293 \\
\hline & $3 \mathrm{MHA}$ & $115 \rightarrow 81$ & 15304 & $115 \rightarrow 73$ & 14281 \\
\hline \multirow[t]{6}{*}{$\begin{array}{l}\text { Analyst } 1 \text {, } \\
\text { measurement } 3 \text { : }\end{array}$} & Compound & $\begin{array}{c}\text { Quantitative } \\
\text { transition }[\mathrm{m} / \mathrm{z}]\end{array}$ & Area & $\begin{array}{c}\text { Qualitative } \\
\text { transition }[\mathrm{m} / \mathrm{z}]\end{array}$ & Area \\
\hline & $d_{10-4 M M P}$ & $109 \rightarrow 46$ & 24312 & $322 \rightarrow 46$ & 7660 \\
\hline & 4MMP & $99 \rightarrow 43$ & 2297 & $312 \rightarrow 43$ & 1314 \\
\hline & $d_{5}-3 M H$ & $138 \rightarrow 85$ & 7789 & $319 \rightarrow 87$ & 2212 \\
\hline & $3 \mathrm{MH}$ & $133 \rightarrow 81$ & 1847 & $314 \rightarrow 82$ & 285 \\
\hline & $3 \mathrm{MHA}$ & $115 \rightarrow 81$ & 15711 & $115 \rightarrow 73$ & 14235 \\
\hline \multirow[t]{6}{*}{$\begin{array}{l}\text { Analyst } 2 \text {, } \\
\text { measurement } 1 \text { : }\end{array}$} & Compound & $\begin{array}{c}\text { Quantitative } \\
\text { transition }[\mathrm{m} / \mathrm{z}]\end{array}$ & Area & $\begin{array}{c}\text { Qualitative } \\
\text { transition }[\mathrm{m} / \mathrm{z}]\end{array}$ & Area \\
\hline & $\overline{d_{10}-4 M M P}$ & $109 \rightarrow 46$ & 23739 & $322 \rightarrow 46$ & 7215 \\
\hline & 4MMP & $99 \rightarrow 43$ & 2462 & $312 \rightarrow 43$ & 1749 \\
\hline & $d_{5}-3 M H$ & $138 \rightarrow 85$ & 6780 & $319 \rightarrow 87$ & 1462 \\
\hline & $3 \mathrm{MH}$ & $133 \rightarrow 81$ & 1654 & $314 \rightarrow 82$ & 235 \\
\hline & $3 \mathrm{MHA}$ & $115 \rightarrow 81$ & 15196 & $115 \rightarrow 73$ & 13618 \\
\hline \multirow[t]{6}{*}{$\begin{array}{l}\text { Analyst } 2 \text {, } \\
\text { measurement 2: }\end{array}$} & Compound & $\begin{array}{c}\text { Quantitative } \\
\text { transition }[\mathrm{m} / \mathrm{z}]\end{array}$ & Area & $\begin{array}{c}\text { Qualitative } \\
\text { transition }[\mathrm{m} / \mathrm{z}]\end{array}$ & Area \\
\hline & $\mathrm{d}_{10-4 M M P}$ & $109 \rightarrow 46$ & 16128 & $322 \rightarrow 46$ & 5262 \\
\hline & $4 \mathrm{MMP}$ & $99 \rightarrow 43$ & 1166 & $312 \rightarrow 43$ & 1131 \\
\hline & $d_{5}-3 M H$ & $138 \rightarrow 85$ & 4888 & $319 \rightarrow 87$ & 1297 \\
\hline & $3 \mathrm{MH}$ & $133 \rightarrow 81$ & 1377 & $314 \rightarrow 82$ & 258 \\
\hline & $3 \mathrm{MHA}$ & $115 \rightarrow 81$ & 12562 & $115 \rightarrow 73$ & 13646 \\
\hline \multirow[t]{6}{*}{$\begin{array}{l}\text { Analyst 2, } \\
\text { measurement } 3 \text { : }\end{array}$} & Compound & $\begin{array}{c}\text { Quantitative } \\
\operatorname{transition}[\mathrm{m} / \mathrm{z}]\end{array}$ & Area & $\begin{array}{c}\text { Qualitative } \\
\operatorname{transition}[\mathrm{m} / \mathrm{z}]\end{array}$ & Area \\
\hline & $\overline{d_{10}-4 M M P}$ & $109 \rightarrow 46$ & 10692 & $322 \rightarrow 46$ & 2866 \\
\hline & 4MMP & $99 \rightarrow 43$ & 1145 & $312 \rightarrow 43$ & 775 \\
\hline & $d_{5}-3 M H$ & $138 \rightarrow 85$ & 4240 & $319 \rightarrow 87$ & 903 \\
\hline & $3 \mathrm{MH}$ & $133 \rightarrow 81$ & 857 & $314 \rightarrow 82$ & 134 \\
\hline & $3 \mathrm{MHA}$ & $115 \rightarrow 81$ & 7281 & $115 \rightarrow 73$ & 6267 \\
\hline
\end{tabular}

